\title{
Rényi Information Divergence via Measure Transformations on Minimal Spanning Trees
}

\author{
Alfred O. Hero ${ }^{1}$ \\ Dept. EECS \\ University of Michigan \\ 1301 Beal Avenue \\ Ann Arbor, MI 48109-2122 USA \\ hero@eecs.umich.edu
}

\author{
Olivier J.J. Michel \\ Laboratoire de Physique, \\ URA-1325 CNRS, \\ École Normale Supérieure de Lyon, \\ 46 allée d'Italie, \\ 69364 Lyon Cedex 07, France \\ omichel@physique.ens-lyon.fr
}

planar $k$-MST approximation of Ravi et al, called the greedy $k$-MST approximation, which runs in polynomial time.

Let $\nu \in(0,1)$ be defined by $\nu=(d-\gamma) / d$ and define the statistic

$$
\hat{H}_{\nu}\left(\mathcal{X}_{n, k}^{*}\right)=\frac{1}{1-\nu} \ln \left(n^{-\nu} L\left(\mathcal{X}_{n, k}^{*}\right)\right)+\beta(\nu, d)
$$
connecting the points converges a.s. in $n$ to the I-divergence after a suitable change of measure.

\section{INTRODUCTION}

Let $\mathcal{X}_{n}=\left\{x_{1}, x_{2}, \ldots, x_{n}\right\}$ denote a sample of i.i.d data points in $R^{d}$ having unknown Lebesgue multivariate density $f(x)$ supported on $[0,1]^{d}$. Define the order $\nu$ Rényi I-divergence [1] with respect to a dominating reference density $f_{o}(x)$

$$
I_{\nu}\left(f, f_{o}\right)=\frac{1}{\nu-1} \ln \int\left(\frac{f(x)}{f_{o}(x)}\right)^{\nu} f_{o}(x) d x
$$

The I-divergence takes on its minimum value (equals zero) if and only if $f=f_{o}$ (a.e.). $I_{\nu}\left(f, f_{o}\right)$ reduces to the Rényi entropy $H_{\nu}(f)$ when $f_{o}$ is equal to a uniform density over $[0,1]^{d}$. Special cases of interest are obtained for $\nu=\frac{1}{2}$ for which one obtains the log Hellinger distance squared and for $\nu \rightarrow 1$ for which one obtains the Kullback-Liebler divergence.

\section{MST's AND ENTROPy Estimation}

A spanning tree $\mathcal{T}$ through the sample $\mathcal{X}_{n}$ is a connected acyclic graph which passes through all the $n$ points $\left\{x_{i}\right\}_{i}$ in the sample. $\mathcal{T}$ is specified by an ordered list of edge (Euclidean) lengths $e_{i j}$ connecting certain pairs $\left(x_{i}, x_{j}\right), i \neq j$, along with a list of edge adjacency relations. The power weighted length of the tree $\mathcal{T}$ is the sum of all edge lengths raised to a power $\gamma \in(0, d)$, denoted by: $\sum_{e \in \mathcal{T}}|e|^{\gamma}$. The minimal spanning tree (MST) is the tree which has the minimal length $L\left(\mathcal{X}_{n}\right)=\min _{\mathcal{T}} \sum_{e \in \mathcal{T}}|e|^{\gamma}$. For any subset $\mathcal{X}_{n, k}$ of $k$ points in $\mathcal{X}_{n}$ define $\mathcal{T}_{\mathcal{X}_{n, k}}$ the $k$-point MST which spans $\mathcal{X}_{n, k}$. The $k$-MST is defined as that $k$-point MST which has minimum length. Thus the $k$-MST spans the densest $k$-dimensional subset $\mathcal{X}_{n, k}^{*}$ of $\mathcal{X}_{n}$. The $k$ MST computation is NP complete. In [2] we presented asymptotic results for a $d$-dimensional extension of the

${ }^{1}$ This research was supported in part by AFOSR under MURI grant F49620-97-0028. where $\beta$ is a constant equal to the $\nu$-th order Rényi entropy of the uniform density on $[0,1]^{d}$. Let $G(x)$ be the coordinate transformation on $[0,1]^{d}$ which maps the reference distribution $f_{o}$ to a uniform distribution and define the transformed data sample $\mathcal{Y}_{n}=G\left(\mathcal{X}_{n}\right)$. Then using the results of [2] it can be shown that $\hat{H}_{\nu}\left(\mathcal{Y}_{n, n}^{*}\right)$ is an a.s. consistent estimator of the I-divergence (1). Furthermore, with $\alpha=k / n, \hat{H}_{\nu}\left(\mathcal{Y}_{n, k}^{*}\right)$ is an $\alpha$-trimmed estimator of I-divergence in the sense that

$\hat{H}_{\nu}\left(\mathcal{Y}_{n, k}^{*}\right) \rightarrow \min _{A: P(A) \geq \alpha} \frac{1}{1-\nu} \ln \int_{A}\left(\frac{f(x)}{f_{o}(x)}\right)^{\nu} f_{o}(x) d x \quad$ (a.s.)

where the minimization is performed over all $d$ dimensional Borel subsets of $[0,1]^{d}$ having probability $P(A)=\int_{A} f_{o}(x) d x \geq \alpha$.

Let $f$ follow the mixture model

$$
f=(1-\epsilon) f_{1}+\epsilon f_{o},
$$

where $f_{o}$ is a known outlier density and $f_{1}, \epsilon \in[0,1]$ are unknown. Then for small $\epsilon$ and $\alpha$ close to one it can easily be shown that the right hand side of (3), which is $I_{\nu}\left(f, f_{o}\right)$, is to a close approximation $I_{\nu}\left(f_{1}, f_{o}\right)$. Thus $\hat{H}_{\nu}\left(\mathcal{Y}_{n, k}^{*}\right)$ is a robust estimator of $I_{\nu}\left(f_{1}, f_{o}\right)$.

Note the following: the estimator $\hat{H}_{\nu}\left(\mathcal{Y}_{n, k}^{*}\right)$ does not require performing the difficult step of density estimation; estimates of various orders $\nu$ of $I_{\nu}$ can be obtained by varying the edge power exponent; the sequence of trees $\mathcal{Y}_{n, 2}, \ldots \mathcal{Y}_{n, n}=\mathcal{Y}_{n}$ provides a natural extension of rank order statistics for multidimensional data. Here $k$ plays the same role as the parameter $\alpha$ in the $\alpha$ trimmed mean estimator for 1-dimensional data.

\section{REFERENCES}

[1] M. Basseville, "Distance measures for signal processing and pattern recognition," Signal Processing, vol. 18, pp. 349-369, 1989

[2] A. Hero and O. Michel, "Asymptotic theory of greedy approximations to minimal k-point random graphs," IEEE Trans. on Inform. Theory, vol. IT-45, no. 6, pp. 1921-1939, Sept. 1999. 